\title{
Construction of Lower and Upper Functions and Their Application to Regular and Singular Periodic Boundary Value Problems
}

\author{
Irena Rachůnková ${ }^{\mathrm{b}, 1}$, Milan Tvrdýa,* \\ ${ }^{a}$ Mathematical Institute, Academy of Sciences of the Czech Republic, \\ 11567 PRAHA 1, Žitná 25, Czech Republic \\ ${ }^{\mathrm{b}}$ Department of Mathematics, Palacký University, 77900 OLOMOUC, \\ Tomkova 40, Czech Republic
}

\begin{abstract}
We present new existence and multiplicity results for regular and singular periodic second order boundary value problems.

Key words: Second order nonlinear ordinary differential equation, periodic solution, singular problem, lower and upper functions, repulsive singularity, Duffing equation.
\end{abstract}

1991 MSC: 34B15, 34C25

\section{Introduction}

In the literature concerning nonlinear boundary value problems we can often find methods based on the lower and upper functions approach (see e.g. [1] and references therein). On the other hand, the problem of construction of lower and upper functions has been solved very rarely. In this paper we fill this gap and present conditions ensuring the existence of nonconstant possibly nonsmooth lower and upper functions to the periodic boundary value problem

$$
u^{\prime \prime}=f(t, u), \quad u(0)=u(2 \pi), \quad u^{\prime}(0)=u^{\prime}(2 \pi)
$$

* Corresponding author.

Email addresses: rachunko@risc.upol.cz (Irena Rachůnková), tvrdy@math.cas.cz (Milan Tvrdý).

1 Supported by the grant No. 201/98/0318 of the Grant Agency of the Czech Republic and by the Council of Czech Government J14/98:153100011 
and find estimates for them. This enables us to prove the existence theorem for the periodic problem for the Duffing equation with a repulsive singularity which extends the classical result of Lazer \& Solimini [4] and supplements the results obtained by some of their followers (see e.g. [2], [8] or [13]). In particular, our result is closely related to that of Omari \& Ye [8].

Throughout the paper we assume:

$f:[0,2 \pi] \times \mathbb{R} \mapsto \mathbb{R}$ fulfils the Carathéodory conditions on $[0,2 \pi] \times \mathbb{R}$, i.e. $f$ has the following properties: (i) for each $x \in \mathbb{R}$ the function $f(., x)$ is measurable on $[0,2 \pi]$; (ii) for almost every $t \in[0,2 \pi]$ the function $f(t,$. is continuous on $\mathbb{R}$; (iii) for each compact set $K \subset \mathbb{R}$ the function $m_{\mathrm{K}}(t)=$ $\sup _{x \in \mathrm{K}}|f(t, x)|$ is Lebesgue integrable on $[0,2 \pi]$. The set of functions satisfying the Carathéodory conditions on $[0,2 \pi] \times \mathbb{R}$ is denoted by $\operatorname{Car}([0,2 \pi] \times \mathbb{R})$.

Furthermore, we keep the following notation:

For a given subinterval $J$ of $\mathbb{R}$ (possibly unbounded), $\mathbb{C}(J)$ denotes the set of functions continuous on $J, \mathbb{L}[0,2 \pi]$ stands for the set of functions (Lebesgue) integrable on $[0,2 \pi], \mathbb{L}_{2}[0,2 \pi]$ is the set of functions square integrable on $[0,2 \pi], \mathbb{A} \mathbb{C}[0,2 \pi]$ denotes the set of functions absolutely continuous on $[0,2 \pi]$ and $\mathbb{B V}[0,2 \pi]$ is the set of functions of bounded variation on $[0,2 \pi]$. If $x \in$ $\mathbb{B V}[0,2 \pi]$ and $t \in[0,2 \pi)$, then the symbol $\Delta^{+} x(t)$ is defined by $\Delta^{+} x(t)=$ $x(t+)-x(t)=\lim _{\tau \rightarrow t+} x(\tau)-x(t)$, while $x^{\text {ac }}$ and $x^{\text {sing }}$ stand for the absolutely continuous part of $x$ and the singular part of $x$, respectively. We suppose $x^{\operatorname{sing}}(0)=0$. For a given $\tau \in[0,2 \pi), \mathbb{A} \mathbb{C}([0,2 \pi] \backslash\{\tau\})$ is the set of functions $x \in \mathbb{B} \mathbb{V}[0,2 \pi]$ such that $x-\Delta^{+} x(\tau) \chi_{(\tau, 2 \pi]} \in \mathbb{A} \mathbb{C}[0,2 \pi]$ (as usual, for $M \subset \mathbb{R}$, $\chi_{M}$ denotes the characteristic function of $\left.M\right)$. For $x \in \mathbb{C}[0,2 \pi], y \in \mathbb{L}[0,2 \pi]$ and $z \in \mathbb{L}_{2}[0,2 \pi]$ we denote $\|x\|_{\infty}=\sup _{t \in[0,2 \pi]}|x(t)|$,

$$
\bar{y}=\frac{1}{2 \pi} \int_{0}^{2 \pi} y(s) \mathrm{d} s,\|y\|_{1}=\int_{0}^{2 \pi}|y(t)| \mathrm{d} t \text { and }\|z\|_{2}=\left(\int_{0}^{2 \pi} z^{2}(t) \mathrm{d} t\right)^{\frac{1}{2}} .
$$

Finally, for $\beta \in \mathbb{L}[0,2 \pi]$, its nonnegative and nonpositive parts are denoted by $\beta^{+}$and $\beta^{-}$, respectively.

By a solution of (1.1) we understand a function $u:[0,2 \pi] \mapsto \mathbb{R}$ such that $u^{\prime} \in \mathbb{A} \mathbb{C}[0,2 \pi], u(0)=u(2 \pi), u^{\prime}(0)=u^{\prime}(2 \pi)$ and $u^{\prime \prime}(t)=f(t, u(t))$ for a.e. $t \in[0,2 \pi]$.

The following definition generalizes most of the earlier ones and is taken from [9].

1.1. Definition. Functions $\left(\sigma_{1}, \rho_{1}\right) \in \mathbb{A} \mathbb{C}[0,2 \pi] \times \mathbb{B} \mathbb{V}[0,2 \pi]$ are said to be lower functions of the problem (1.1), if the singular part $\rho_{1}^{\text {sing }}$ of $\rho_{1}$ is nondecreasing on $[0,2 \pi], \sigma_{1}^{\prime}(t)=\rho_{1}(t)$ and $\rho_{1}^{\prime}(t) \geq f\left(t, \sigma_{1}(t)\right)$ for a.e. $t \in[0,2 \pi]$, $\sigma_{1}(0)=\sigma_{1}(2 \pi)$ and $\rho_{1}(0)=\rho_{1}(2 \pi)$.

Similarly, functions $\left(\sigma_{2}, \rho_{2}\right) \in \mathbb{A} \mathbb{C}[0,2 \pi] \times \mathbb{B} \mathbb{V}[0,2 \pi]$ are said to be upper func- 
tions of (1.1), if $\rho_{2}^{\text {sing }}$ is nonincreasing on $[0,2 \pi], \sigma_{2}^{\prime}(t)=\rho_{2}(t)$ and $\rho_{2}^{\prime}(t) \leq$ $f\left(t, \sigma_{2}(t)\right)$ for a.e. $t \in[0,2 \pi], \sigma_{2}(0)=\sigma_{2}(2 \pi)$ and $\rho_{2}(0)=\rho_{2}(2 \pi)$.

For the existence results obtained in this paper we will need the following theorem which is contained in [9, Theorems 4.1 and 4.2].

1.2. Theorem. Let $\left(\sigma_{1}, \rho_{1}\right)$ and $\left(\sigma_{2}, \rho_{2}\right)$ be respectively lower and upper functions of (1.1).

(I) Suppose $\sigma_{1}(t) \leq \sigma_{2}(t)$ on $[0,2 \pi]$. Then there is a solution $u$ of $(1.1)$ such that $\sigma_{1}(t) \leq u(t) \leq \sigma_{2}(t)$ on $[0,2 \pi]$.

(II) Suppose $\sigma_{1}(t) \geq \sigma_{2}(t)$ on $[0,2 \pi]$ and there is $m \in \mathbb{L}[0,2 \pi]$ such that $f(t, x) \geq m(t) \quad$ or $f(t, x) \leq m(t))$ for a.e. $t \in[0,2 \pi]$ and all $x \in \mathbb{R}$. Then there are a solution $u$ of (1.1) and $t_{u} \in[0,2 \pi]$ such that $\sigma_{2}\left(t_{u}\right) \leq u\left(t_{u}\right) \leq \sigma_{1}\left(t_{u}\right)$ and $\left\|u^{\prime}\right\|_{\infty} \leq\|m\|_{1}$.

\section{Construction of lower and upper functions}

Let us consider an auxiliary boundary value problem

$$
\begin{gathered}
\sigma^{\prime}=\rho, \quad \rho^{\prime}=\beta(t), \\
\sigma(0)=\sigma(2 \pi)=c, \rho(0)=\rho(2 \pi), \Delta^{+} \rho(\tau)=-2 \pi \bar{\beta},
\end{gathered}
$$

where $\beta \in \mathbb{L}[0,2 \pi]$ and $c \in \mathbb{R}$.

2.1. Definition. Let $\tau \in[0,2 \pi), c \in \mathbb{R}$ and $\beta \in \mathbb{L}[0,2 \pi]$ be given. By a solution of the problem (2.1), (2.2) we mean a couple of functions $(\sigma, \rho) \in$ $\mathbb{A} \mathbb{C}[0,2 \pi] \times \mathbb{A} \mathbb{C}([0,2 \pi] \backslash\{\tau\})$ satisfying (2.2) and

$$
\sigma^{\prime}(t)=\rho(t), \quad \rho^{\prime}(t)=\beta(t) \quad \text { a.e. on }[0,2 \pi] .
$$

2.2. Proposition. Let $c \in \mathbb{R}, \tau \in[0,2 \pi)$ and $\beta \in \mathbb{L}[0,2 \pi]$. Then the problem (2.1), (2.2) possesses a unique solution $(\sigma, \rho)$. Moreover, $\rho^{\text {sing }}=-2 \pi \bar{\beta} \chi_{(\tau, 2 \pi]}$ and

where

$$
\sigma(t)=c-g(t, \tau)(2 \pi \bar{\beta})+\int_{0}^{2 \pi} g(t, s) \beta(s) \mathrm{d} s \text { on }[0,2 \pi]
$$

$$
g(t, s)=\frac{t(s-2 \pi)}{2 \pi} \text { if } t \leq s \quad \text { and } \quad g(t, s)=\frac{(t-2 \pi) s}{2 \pi} \text { if } s<t .
$$

Proof. For $c, c_{1} \in \mathbb{R}$, put

$$
\sigma(t)=\left\{\begin{array}{cl}
c+c_{1} t+\int_{0}^{t}(t-s) \beta(s) \mathrm{d} s & \text { if } t \leq \tau, \\
c+c_{1}(t-2 \pi)-\int_{t}^{2 \pi}(t-s) \beta(s) \mathrm{d} s & \text { if } \tau<t
\end{array}\right.
$$


and

$$
\rho(t)=\left\{\begin{array}{l}
c_{1}+\int_{0}^{t} \beta(s) \mathrm{d} s \text { if } t \leq \tau, \\
c_{1}-\int_{t}^{2 \pi} \beta(s) \mathrm{d} s \text { if } \tau<t .
\end{array}\right.
$$

Then $\sigma$ and $\rho$ belong to $\mathbb{A C}([0,2 \pi] \backslash\{\tau\})$ and satisfy (2.3) and (2.2). Furthermore, $\Delta^{+} \sigma(\tau)=0$ (i.e. $\sigma$ is absolutely continuous on $[0,2 \pi]$ ) if and only if

$$
c_{1}=-\int_{0}^{2 \pi} \frac{\tau-s}{2 \pi} \beta(s) \mathrm{d} s,
$$

while $c \in \mathbb{R}$ may be arbitrary. Inserting (2.8) into (2.6) we can check that $\sigma$ verifies (2.4). Finally, in virtue of (2.7) we have

$$
\rho(t)+2 \pi \bar{\beta} \chi_{(\tau, 2 \pi]}(t)=c_{1}+\int_{0}^{t} \beta(s) \mathrm{d} s \quad \text { on } \quad[0,2 \pi]
$$

i.e. $\rho^{\mathrm{ac}}=\rho+2 \pi \bar{\beta} \chi_{(\tau, 2 \pi]}$ and $\rho^{\mathrm{sing}}=-2 \pi \bar{\beta} \chi_{(\tau, 2 \pi]}$.

The following lemma will be often used in this paper.

2.3. Lemma. Let $u \in \mathbb{A} \mathbb{C}[0,2 \pi]$ and $u^{\prime} \in \mathbb{L}_{2}[0,2 \pi]$. Then

$$
\|u\|_{2} \leq 2\left\|u^{\prime}\right\|_{2}
$$

holds whenever $u(0)=u(2 \pi)=0$ and

$$
\|u\|_{\infty} \leq \sqrt{\frac{\pi}{6}}\left\|u^{\prime}\right\|_{2}
$$

is true whenever $u(0)=u(2 \pi)$ and $\bar{u}=0$.

Proof. The inequality (2.9) is due to Scheeffer [12, p. 207] (see also [7, II.2]). For the inequality (2.10) (Sobolev's inequality) see e.g. [6, Proposition 1.3]).

2.4. Proposition. Assume that there are $a, A \in \mathbb{R}, \tau \in[0,2 \pi)$ and $b \in$ $\mathbb{L}[0,2 \pi]$ such that $a \leq 0, \bar{b}=0$ and

$$
f(t, x) \leq a+b(t) \text { for a.e. } t \in[0,2 \pi] \text { and all } x \in[A(t), B(t)],
$$

where

$$
\begin{gathered}
A(t)=A+a h(t, \tau), B(t)=A(t)+\frac{\pi}{3}\|b\|_{1} \quad \text { on }[0,2 \pi], \\
h(t, \tau)=\frac{t(2 \pi-2 \tau+t)}{2} \text { if } t \leq \tau, h(t, \tau)=\frac{(2 \pi-t)(2 \tau-t)}{2} \text { if } \tau \leq t .
\end{gathered}
$$


Then there exist lower functions $(\sigma, \rho)$ of (1.1) such that

$$
A(t) \leq \sigma(t) \leq B(t) \text { on }[0,2 \pi] \quad \text { and } \quad \rho \in \mathbb{A} \mathbb{C}([0,2 \pi] \backslash\{\tau\}) .
$$

Proof. By Proposition 2.2, the problem (2.1), (2.2) with $\beta(t)=a+b(t)$ a.e. on $[0,2 \pi]$ has a unique solution $(\sigma, \rho)$ for any $c \in \mathbb{R}$. In particular, $\rho \in \mathbb{A} \mathbb{C}([0,2 \pi] \backslash$ $\{\tau\})$. Moreover, with respect to (2.4), (2.5) and (2.13), $\sigma$ has the form

$$
\begin{aligned}
\sigma(t) & =c-2 \pi a g(t, \tau)+\frac{a}{2} t(t-2 \pi)+\int_{0}^{2 \pi} g(t, s) b(s) \mathrm{d} s \\
& =c+a h(t, \tau)+\int_{0}^{2 \pi} g(t, s) b(s) \mathrm{d} s \quad \text { on }[0,2 \pi] .
\end{aligned}
$$

Let us put

and

$$
c_{0}=-\frac{1}{2 \pi} \int_{0}^{2 \pi}\left(\int_{0}^{2 \pi} g(t, s) b(s) \mathrm{d} s\right) \mathrm{d} t
$$

$$
\sigma_{0}(t)=c_{0}+\int_{0}^{2 \pi} g(t, s) b(s) \mathrm{d} s \quad \text { for } t \in[0,2 \pi] .
$$

Then $\overline{\sigma_{0}}=0$ and $\sigma_{0}^{\prime \prime}(t)=b(t)$ a.e. on $[0,2 \pi]$. Multiplying the last relation by $\sigma_{0}$, integrating it over $[0,2 \pi]$ and using the Hölder inequality we get $\left\|\sigma_{0}^{\prime}\right\|_{2}^{2} \leq\|b\|_{1}\left\|\sigma_{0}\right\|_{\infty}$. Further, the Sobolev inequality (2.10) (see Lemma 2.3) yields $\left\|\sigma_{0}^{\prime}\right\|_{2}^{2} \leq \sqrt{\frac{\pi}{6}}\|b\|_{1}\left\|\sigma_{0}^{\prime}\right\|_{2}$, and so $\left\|\sigma_{0}^{\prime}\right\|_{2} \leq \sqrt{\frac{\pi}{6}}\|b\|_{1}$, wherefrom using again the Sobolev inequality (2.10) we get

$$
\left\|\sigma_{0}\right\|_{\infty} \leq \frac{\pi}{6}\|b\|_{1}
$$

This implies

$$
a h(t, \tau)-\frac{\pi}{6}\|b\|_{1} \leq a h(t, \tau)+\sigma_{0}(t) \leq a h(t, \tau)+\frac{\pi}{6}\|b\|_{1} \text { on }[0,2 \pi] .
$$

Now, choosing $c=c_{0}+\frac{\pi}{6}\|b\|_{1}+A$, we obtain $\sigma(t)=\frac{\pi}{6}\|b\|_{1}+A+a h(t, \tau)+\sigma_{0}(t)$ on $[0,2 \pi]$. Thus, with respect to $(2.15)$, we have

$$
A+a h(t, \tau) \leq \sigma(t) \leq A+a h(t, \tau)+\frac{\pi}{3}\|b\|_{1} \text { on }[0,2 \pi],
$$

which means that (2.14) holds. Since $a \leq 0$, according to (2.11) this yields

$$
\rho^{\prime}(t)=a+b(t) \geq f(t, \sigma(t)) \quad \text { a.e. on }[0,2 \pi] .
$$

Furthermore, with respect to $(2.2)$ we have $\sigma(0)=\sigma(2 \pi)$ and $\rho(0)=\rho(2 \pi)$ and hence, by Definition 1.1 the functions $(\sigma, \rho)$ are lower functions of (1.1).

The following assertion is dual to Proposition 2.4 and its proof can be omitted. 
2.5. Proposition. Assume that there are $a, A \in \mathbb{R}, \tau \in[0,2 \pi)$ and $b \in$ $\mathbb{L}[0,2 \pi]$ such that $a \geq 0, \bar{b}=0$,

$$
f(t, x) \geq a+b(t) \text { for a.e. } t \in[0,2 \pi] \text { and all } x \in[A(t), B(t)],
$$

where $A(t)$ and $B(t)$ are defined by (2.12) and (2.13). Then there exist upper functions $(\sigma, \rho)$ of (1.1) fulfilling (2.14).

Theorems 2.6 and 2.7 are simple examples of existence results which follow immediately from Theorem 1.2 and Propositions 2.4 and 2.5.

2.6. Theorem. Assume that there are $A_{1}, A_{2} \in \mathbb{R}, a_{1} \leq 0, a_{2} \geq 0, \tau_{1}, \tau_{2} \in$ $[0,2 \pi]$ and $b_{1}, b_{2} \in \mathbb{L}[0,2 \pi]$ such that $\overline{b_{1}}=\overline{b_{2}}=0$,

$$
(-1)^{i} f(t, x) \geq(-1)^{i}\left(a_{i}+b_{i}(t)\right)
$$

for all $x \in\left[A_{i}+a_{i} h\left(t, \tau_{i}\right), B_{i}+a_{i} h\left(t, \tau_{i}\right)\right]$, a.e. $t \in[0,2 \pi]$ and all $i \in\{1,2\}$,

$$
A_{2}+a_{2} h\left(t, \tau_{2}\right) \geq B_{1}+a_{1} h\left(t, \tau_{1}\right) \quad \text { for all } t \in[0,2 \pi],
$$

where $h(t, \tau)$ is given by (2.13) and $B_{i}-A_{i}=\frac{\pi}{3}\left\|b_{i}\right\|_{1}$ for $i=1,2$. Then the problem (1.1) possesses a solution $u$ such that

$$
A_{1}+a_{1} h\left(t, \tau_{1}\right) \leq u(t) \leq B_{2}+a_{2} h\left(t, \tau_{2}\right) \quad \text { on }[0,2 \pi] .
$$

2.7. Theorem. Assume that there are $A_{1}, A_{2} \in \mathbb{R}, a_{1} \leq 0, a_{2} \geq 0, \tau_{1}, \tau_{2} \in$ $[0,2 \pi]$ and $b_{1}, b_{2} \in \mathbb{L}[0,2 \pi]$ such that the assumptions of Theorem 2.6 are satisfied with

$$
A_{1}+a_{1} h\left(t, \tau_{1}\right) \geq B_{2}+a_{2} h\left(t, \tau_{2}\right) \quad \text { for all } t \in[0,2 \pi]
$$

instead of (2.16). Furthermore, let there exist $m \in \mathbb{L}[0,2 \pi]$ such that $f(t, x) \geq$ $m(t)($ or $f(t, x) \leq m(t))$ for a.e. $t \in[0,2 \pi]$ and all $x \in \mathbb{R}$. Then the problem (1.1) possesses a solution $u$ such that $\left\|u^{\prime}\right\|_{\infty} \leq\|m\|_{1}$ and $A_{2}+a_{2} h\left(t_{u}, \tau_{2}\right) \leq$ $u\left(t_{u}\right) \leq B_{1}+a_{1} h\left(t_{u}, \tau_{1}\right)$ for some $t_{u} \in[0,2 \pi]$.

\section{Periodic problems with strong singularity}

We will consider the following singular Duffing equation with periodic conditions

$$
u^{\prime \prime}-g(u)=e(t), \quad u(0)=u(2 \pi), \quad u^{\prime}(0)=u^{\prime}(2 \pi)
$$

where

$$
g \in \mathbb{C}(0, \infty) \quad \text { and } \quad e \in \mathbb{L}[0,2 \pi]
$$


and $g$ has strong singularity at 0 , i.e.

$$
\lim _{x \rightarrow 0+} \int_{x}^{1} g(\xi) \mathrm{d} \xi=\infty
$$

Classical Lazer and Solimini's considerations [4] concerning the problem (3.1) have been extended by several authors (see e.g. [2], [3], [5], [8], [10], [11] and [13]). Provided $g \in \mathbb{C}(0, \infty), e$ is essentially bounded on $[0,2 \pi]$ and under the assumptions (3.3),

$$
\begin{aligned}
& \lim _{x \rightarrow 0+} g(x)=\infty, \\
& \liminf _{x \rightarrow \infty} \frac{g(x)}{x} \geq-\frac{1}{4}, \quad \liminf _{x \rightarrow \infty} \frac{1}{x^{2}} \int_{1}^{x} g(\xi) \mathrm{d} \xi>-\frac{1}{8}, \\
& \text { there is } d>0 \text { such that } g(x) \leq-\bar{e} \text { for all } x \in[d, \infty),
\end{aligned}
$$

Omari and Ye proved the existence of a solution to (3.1) in [8, Theorem 1.2]. In [10, Theorem 3.2] we showed a related result, where $e$ need not be essentially bounded and (3.4) need not be fulfilled. Here we generalize the result of [10] for functions $g$ unbounded from below.

3.1. Theorem. Assume (3.2), (3.3),

$$
\liminf _{x \rightarrow 0+} g(x)>-\infty
$$

and

$$
\liminf _{x \rightarrow \infty} \frac{g(x)}{x}>-\frac{1}{4}
$$

Furthermore, let there exist $A_{1}, A_{2} \in(0, \infty)$ such that

$$
\begin{aligned}
& g(x) \leq-\bar{e} \quad \text { for all } x \in\left[A_{1}, B_{1}\right] \\
& g(x) \geq-\bar{e} \quad \text { for all } x \in\left[A_{2}, B_{2}\right]
\end{aligned}
$$

where $A_{1} \geq B_{2}$ and

$$
B_{1}-A_{1}=B_{2}-A_{2}=\frac{\pi}{3}\|e-\bar{e}\|_{1} .
$$

Then the problem (3.1) has a positive solution.

3.2. Remark. If $g \in \mathbb{C}(0, \infty)$ satisfies $(3.3)$, then $\lim \sup _{x \rightarrow 0+} g(x)=\infty$, which implies the existence of a sequence $\left\{\varepsilon_{n}\right\}_{n=1}^{\infty} \subset(0,1)$ such that

$$
g\left(\varepsilon_{n}\right)>0 \quad \text { for all } n \in \mathbb{N}, \quad \lim _{n} \varepsilon_{n}=0, \quad \lim _{n} g\left(\varepsilon_{n}\right)=\infty .
$$

For the proof of Theorem 3.1 we will need the following two lemmas, where 
we deal with the auxiliary family of problems

$$
u^{\prime \prime}=g_{n}(u)+e(t), \quad u(0)=u(2 \pi), \quad u^{\prime}(0)=u^{\prime}(2 \pi),
$$

where $n \in \mathbb{N}, \varepsilon_{n}$ are from (3.10) and

$$
g_{n}(x)=\left\{\begin{array}{cl}
0 & \text { if } x<0, \\
g\left(\varepsilon_{n}\right) \frac{x}{\varepsilon_{n}} & \text { if } x \in\left[0, \varepsilon_{n}\right], \\
g(x) & \text { if } x>\varepsilon_{n} .
\end{array}\right.
$$

3.3. Lemma. Assume that $g \in \mathbb{C}(0, \infty)$ satisfies (3.3), (3.5) and (3.6) and let $g_{n}, n \in \mathbb{N}$, be given by (3.12). Then there exist $\eta \in\left(0, \frac{1}{4}\right)$ and $C \geq 0$ such that

$$
g_{n}(x) x \geq-\left(\frac{1}{4}-\eta\right) x^{2}-C|x| \quad \text { for all } x \in \mathbb{R} \text { and all } n \in \mathbb{N} .
$$

Proof. By (3.6), there are $\eta \in\left(0, \frac{1}{4}\right)$ and $A \in(1, \infty)$ such that

$$
\frac{g(x)}{x} \geq-\left(\frac{1}{4}-\eta\right) \text { for all } x \geq A
$$

Put

$$
p(x)=\left\{\begin{array}{cl}
0 & \text { if } x<0 \\
g(A) \frac{x}{A} & \text { if } x \in[0, A] \\
g(x) & \text { if } x>A
\end{array}\right.
$$

and $q_{n}(x)=g_{n}(x)-p(x)$ on $\mathbb{R}$. In virtue of (3.5), there is $C \geq 0$ such that $q_{n}(x) \geq-C$ for all $x \in \mathbb{R}$ and all $n \in \mathbb{N}$. Thus, since according to (3.14) and (3.15) we also have

$$
p(x) \geq-\left(\frac{1}{4}-\eta\right) x \quad \text { for all } x \in[0, \infty)
$$

we deduce that (3.13) is true.

3.4. Lemma. Assume that $g$ and $g_{n}, n \in \mathbb{N}$, are as in Lemma 3.3. Then for any $r>0$ and any $e \in \mathbb{L}[0,2 \pi]$ there exists $R \geq r$ such that

$$
u(t) \leq R \quad \text { on }[0,2 \pi]
$$

holds for all $n \in \mathbb{N}$ and all solutions $u$ of (3.11) with the property

$$
\min _{t \in[0,2 \pi]} u(t) \leq r
$$


Proof. Assume that (3.16) does not hold. Then we can choose a subsequence $\left\{g_{k}\right\}_{k=1}^{\infty}$ of the sequence $\left\{g_{n}\right\}_{n=1}^{\infty}$ and a sequence of solutions $\left\{u_{k}\right\}_{k=1}^{\infty}$ of the corresponding problems (3.11) satisying (3.17) and

$$
\lim _{k} \max _{t \in[0,2 \pi]} u_{k}(t)=\infty
$$

In particular, for any $k \in \mathbb{N}$, there is $t_{k} \in[0,2 \pi]$ such that $u_{k}\left(t_{k}\right)=r$. Furthermore, if we extend the functions $u_{k}, k \in \mathbb{N}$, and $e$ to functions $2 \pi$ periodic on $\mathbb{R}$, we get that

$$
u_{k}^{\prime \prime}(t)=g_{k}\left(u_{k}(t)\right)+e(t) \text { for a.e. } t \in \mathbb{R} \text { and any } k \in \mathbb{N} \text {. }
$$

On the other hand, if we multiply (3.19) by $u_{k}(t)$, integrate from $t_{k}$ to $t_{k}+2 \pi$ and take into account Lemma 3.3, we get that there exist $\eta \in\left(0, \frac{1}{4}\right)$ and $C \geq 0$ such that for any $k \in \mathbb{N}$

$$
\begin{aligned}
\left\|u_{k}^{\prime}\right\|_{2}^{2} & =-\int_{t_{k}}^{t_{k}+2 \pi} g_{k}\left(u_{k}(s)\right) u_{k}(s) \mathrm{d} s-\int_{t_{k}}^{t_{k}+2 \pi} e(s) u_{k}(s) \mathrm{d} s \\
& \leq\left(\frac{1}{4}-\eta\right)\left\|u_{k}\right\|_{2}^{2}+C\left\|u_{k}\right\|_{1}+\|e\|_{1}\left\|u_{k}\right\|_{\infty}
\end{aligned}
$$

holds. Furthermore,

$$
\left\|u_{k}\right\|_{\infty} \leq\left|u_{k}\left(t_{k}\right)\right|+\int_{t_{k}}^{t_{k}+2 \pi}\left|u_{k}^{\prime}(s)\right| \mathrm{d} s \leq r+\sqrt{2 \pi}\left\|u_{k}^{\prime}\right\|_{2} .
$$

Thus,

$$
\left(\left\|u_{k}^{\prime}\right\|_{2}-\|e\|_{1} \sqrt{\frac{\pi}{2}}\right)^{2} \leq\left(\frac{1}{4}-\eta\right)\left\|u_{k}\right\|_{2}^{2}+\sqrt{2 \pi} C\left\|u_{k}\right\|_{2}+\|e\|_{1} r+\frac{\pi}{2}\|e\|_{1}^{2} .
$$

Inserting $u_{k}(t) \equiv v_{k}(t)+r$ on $\mathbb{R}$ into (3.21), we obtain

$$
\frac{\left(\left\|v_{k}^{\prime}\right\|_{2}-c\right)^{2}}{\left\|v_{k}\right\|_{2}^{2}} \leq \frac{1}{4}-\eta+\frac{a}{\left\|v_{k}\right\|_{2}}+\frac{b}{\left\|v_{k}\right\|_{2}^{2}},
$$

where $a, b, c \in \mathbb{R}$ do not depend on $k$. Now, (3.18), (3.20) and (3.21) yield

$$
\lim _{k}\left\|v_{k}^{\prime}\right\|_{2}=\infty \quad \text { and } \quad \lim _{k}\left\|v_{k}\right\|_{2}=\infty
$$

Since $v_{k}\left(t_{k}\right)=v\left(t_{k}+2 \pi\right)=0$, by Scheeffer's inequality (2.9) we have $\left\|v_{k}\right\|_{2}^{2} \leq$ $4\left\|v_{k}^{\prime}\right\|_{2}^{2}$ and

$$
\frac{\left(\left\|v_{k}^{\prime}\right\|_{2}-c\right)^{2}}{\left\|v_{k}\right\|_{2}^{2}} \geq \frac{\left(\left\|v_{k}^{\prime}\right\|_{2}-c\right)^{2}}{4\left\|v_{k}^{\prime}\right\|_{2}^{2}}
$$


Therefore by virtue of (3.22) and (3.23) we have

$$
\frac{1}{4}=\lim _{k} \frac{\left(\left\|v_{k}^{\prime}\right\|_{2}-c\right)^{2}}{4\left\|v_{k}^{\prime}\right\|_{2}^{2}} \leq \lim _{k}\left(\frac{1}{4}-\eta+\frac{a}{\left\|v_{k}\right\|_{2}}+\frac{b}{\left\|v_{k}\right\|_{2}^{2}}\right)=\frac{1}{4}-\eta,
$$

a contradiction.

Proof of Theorem 3.1. Let $A>1$ and $R \geq A$ be given by (3.14) and Lemma 3.4 with $r=B_{1}$, respectively. In virtue of (3.2) and (3.5) we have $g_{*}:=\min \left\{0, \inf _{x \in(0, R]} g(x)\right\} \in \mathbb{R}$. Put

$$
K=\|e\|_{1}+2 \pi\left|g_{*}\right| \quad \text { and } \quad K^{*}=K\|e\|_{1}+\int_{A_{2}}^{R}|g(x)| \mathrm{d} x .
$$

It follows from (3.3) and Remark 3.2 that we can choose $\varepsilon \in\left\{\varepsilon_{n}\right\}_{n=1}^{\infty}$ such that $\varepsilon \in\left(0, A_{2}\right)$ and

$$
\int_{\varepsilon}^{A_{2}} g(x) \mathrm{d} x>K^{*} \text { and } g(\varepsilon)>0
$$

For $x \in \mathbb{R}$ and a.e. $t \in[0,2 \pi]$, put $f(t, x)=e(t)+\widetilde{g}(x)$, where

$$
\widetilde{g}(x)=\left\{\begin{array}{cl}
0 & \text { if } x<0, \\
g(\varepsilon) \frac{x}{\varepsilon} & \text { if } x \in[0, \varepsilon), \\
g(x) & \text { if } x \in[\varepsilon, R), \\
g(R) & \text { if } x \geq R .
\end{array}\right.
$$

Then $f \in \operatorname{Car}([0,2 \pi] \times \mathbb{R})$ fulfils the assumptions of Theorem 2.7 with $a_{1}=$ $a_{2}=0, b_{1}(t)=b_{2}(t)=e(t)-\bar{e}$ a.e. on $[0,2 \pi]$ and $m(t)=g_{*}+e(t)$ a.e. on $[0,2 \pi]$. Thus, by Theorem 2.7, the problem (1.1) has a solution $u$ such that $u\left(t_{u}\right) \in\left[A_{2}, B_{1}\right]$ for some $t_{u} \in[0,2 \pi]$ and $\left\|u^{\prime}\right\|_{\infty} \leq K$. By Lemma 3.4 we have $u(t) \leq R$ for all $t \in[0,2 \pi]$. It remains to show that $u(t) \geq \varepsilon$ holds on $[0,2 \pi]$.

Let $t_{0}, t_{1} \in[0,2 \pi]$ and $u\left(t_{0}\right)=\min _{t \in[0,2 \pi]} u(t)$ and $u\left(t_{1}\right)=\max _{t \in[0,2 \pi]} u(t)$. Clearly, $A_{2} \leq u\left(t_{1}\right) \leq R$. Due to the periodic boundary conditions we have $u^{\prime}\left(t_{0}\right)=u^{\prime}\left(t_{1}\right)=0$. Now, multiplying the differential relation $u^{\prime \prime}(t)=e(t)+$ $\widetilde{g}(u(t))$ by $u^{\prime}(t)$ and integrating over $\left[t_{0}, t_{1}\right]$, we get

$$
0=\int_{t_{0}}^{t_{1}} u^{\prime \prime}(t) u^{\prime}(t) \mathrm{d} t=\int_{t_{0}}^{t_{1}} e(t) u^{\prime}(t) \mathrm{d} t+\int_{t_{0}}^{t_{1}} \tilde{g}(u(t)) u^{\prime}(t) \mathrm{d} t
$$

i.e.

$$
\int_{u\left(t_{0}\right)}^{u\left(t_{1}\right)} \widetilde{g}(x) \mathrm{d} x=-\int_{t_{0}}^{t_{1}} e(t) u^{\prime}(t) \mathrm{d} t \leq K\|e\|_{1} .
$$


Further,

$$
\int_{u\left(t_{0}\right)}^{A_{2}} \widetilde{g}(x) \mathrm{d} x \leq K\|e\|_{1}+\int_{A_{2}}^{R}|\widetilde{g}(x)| \mathrm{d} x=K^{*}
$$

which, with respect to (3.24), is possible only if $u\left(t_{0}\right) \geq \varepsilon$. Thus, $u$ is a solution to $(3.1)$.

3.5. Theorem. If $e$ is essentially bounded from below on $[0,2 \pi]$, then the condition (3.8) can be omitted in Theorem 3.1.

Proof. By (3.10) there is $A_{2} \in\left(0, A_{1}\right)$ such that $e(t)+g\left(A_{2}\right) \geq 0$ a.e. on $[0,2 \pi]$. Taking this $A_{2}$ we can argue as in the proof of Theorem 3.1 with the only difference that $b_{2}(t)=0$ a.e. on $[0,2 \pi]$, which implies $A_{2}=B_{2}$ in the application of Theorem 2.7.

Let us complete the above existence results by an easy consequence of Theorem 2.6.

3.6. Theorem. Assume that (3.2), (3.7), (3.8) and (3.9) are satisfied and let $A_{2} \geq B_{1}$. Then (3.1) has a solution $u$ such that $A_{1} \leq u(t) \leq B_{2}$ on $[0,2 \pi]$.

Proof. For a.e. $t \in[0,2 \pi]$ define $f(t, x)=e(t)+g\left(A_{1}\right)$ if $x<A_{1}$ and $f(t, x)=$ $e(t)+g(x)$ if $x \geq A_{1}$. Then $f \in \operatorname{Car}([0,2 \pi] \times \mathbb{R})$ fulfils the assumptions of Theorem 2.6 with $a_{1}=a_{2}=0, b_{1}(t)=b_{2}(t)=e(t)-\bar{e}$ a.e. on $[0,2 \pi]$. Hence, by Theorem 2.6, the problem (1.1) has a solution $u$ satisfying $A_{1} \leq u(t) \leq B_{2}$ on $[0,2 \pi]$, which means that $u$ is a solution of $(3.1)$.

3.7. Example. Notice that the function

$$
g(x)=-0.24 x+\frac{1+\sin \left(\frac{\pi}{x}\right)}{x}, \quad x \in(0, \infty),
$$

verifies the assumptions (3.2), (3.3), (3.5) and (3.6) of Theorem 3.1, while it does not satisfy the condition (3.4) required by Omari and Ye in $[8$, Theorem 1.2]. Since $\lim _{x \rightarrow \infty} g(x)=-\infty$, we can find for any $e \in \mathbb{L}[0,2 \pi]$ and any $B_{2}>0$ a number $A_{1} \in\left[B_{2}, \infty\right)$ such that $g$ fulfils (3.7) with any $B_{1} \in\left(A_{1}, \infty\right)$. Thus, by Theorem 3.5, if $e$ is essentially bounded from below, the problem (3.1) with $g$ given by (3.25) has at least one positive solution. Provided $e$ is not essentially bounded from below, we will use Theorem 3.1 which requires that $g$ fulfil (3.8). Let us restrict ourselves to $e \in \mathbb{L}[0,2 \pi]$ such that $\bar{e}=-7$. We can show that the equation $g(x)=7$ has exactly 5 roots $x_{i}, i=1,2, \ldots, 5$, in the interval $[0.12, \infty)$. (In particular, we have $x_{1} \approx 0.126, x_{2} \approx 0.143, x_{3} \approx$ $0.165, x_{4} \approx 0.206, x_{5} \approx 0.236, g(x)>7$ on $\left(x_{2}, x_{3}\right) \cup\left(x_{4}, x_{5}\right)$ and $g(x)<7$ on $\left(x_{1}, x_{2}\right) \cup\left(x_{3}, x_{4}\right) \cup\left(x_{5}, \infty\right)$.) Let $2 d<x_{3}-x_{2}$ and assume in addition that $\pi\|e-\bar{e}\|_{\mathbb{L}} \leq 3 d$. We have $x_{2}-x_{1}>d$ and $x_{i+1}-x_{i}>2 d$ for $i=2,3,4$. We 
can apply Theorem 3.6 to obtain the existence of solutions $u_{1}$ and $u_{2}$ of the problem (3.1) such that $u_{1}(t) \in\left[x_{2}-d, x_{2}+d\right]$ and $u_{2}(t) \in\left[x_{4}-d, x_{4}+d\right]$ on $t \in[0,2 \pi]$, i.e. $u_{1}(t)<u_{2}(t)$ on $[0,2 \pi]$. Moreover, by Theorem 3.1 there is another solution $u_{3}$ of (3.1) such that $u_{3}\left(t_{3}\right) \in\left[x_{3}-d, x_{3}+d\right]$ for some $t_{3} \in[0,2 \pi]$. In virtue of the definition of $d$ this means that $u_{3}$ can coincide neither with $u_{1}$ nor with $u_{2}$.

3.8. Remark. The existence theorem which concerns the case $A_{1} \geq B_{2}$ and does not need (3.3) has been proved in [11, Corollary 3.7].

\section{References}

[1] C. De Coster, P. Habets. Lower and upper solutions in the theory of ODE boundary value problems: Classical and recent results. F. Zanolin (ed.), Nonlinear Analysis and Boundary Value Problems for Ordinary Differential Equations. Wien: Springer. CISM Courses Lect. 371 (1996), 1-78.

[2] M. Del Pino, R. Manásevich and A. Montero. T-periodic solutions for some second order differential equations with singularities. Proc. Royal Soc. Edinburgh 120A (1992), 231-244.

[3] P. Habets and L. Sanchez. Periodic solutions of some Liénard equations with singularities. Proc. Amer. Math. Soc. 109 (1990), 1035-1044.

[4] A. C. LAzER AND S. Solimini. On periodic solutions of nonlinear differential equations with singularities. Proc. Amer. Math. Soc. 99 (1987), 109-114.

[5] J. Mawhin. Topological degree and boundary value problems for nonlinear differential equations. M. Furi (ed.), Topological methods for ordinary differential equations. Berlin: Springer-Verlag, Lect. Notes Math. 1537 (1993), $73-142$

[6] J. Mawhin and M. Willem. Critical Point Theory and Hamiltonian Systems. Applied Mathematical Sciences 74, Springer-Verlag, Berlin, 1989.

[7] D. S. Mitrinović, J. E. PeČARIĆ And A. M. Fink. Inequalities Involving Functions and Their Integrals and Derivatives. Kluwer, Dordrecht, 1991.

[8] P. Omari and W. YE. Necessary and sufficient conditions for the existence of periodic solutions of second order ordinary differential equations with singular nonlinearities. Differential Integral Equations 8 (1995), 1843-1858.

[9] I. RACHŮNKOVÁ AND M. TVRDÝ. Nonlinear systems of differential inequalities and solvability of certain nonlinear second order boundary value problems. $J$. Inequal. Appl., to appear.

[10] I. RACHŮNKOVÁ AND M. TVRDÝ. Method of lower and upper functions and the existence of solutions to singular periodic problems for second order nonlinear differential equations. Math. Notes Miskolc, to appear.

[11] I. RACHŮNKOVÁ, M. TVRDÝ AND I. VRKOČ. Existence of nonnegative and nonpositive solutions for second order periodic boundary value problems. $J$. Differential Equations, to appear.

[12] L. Scheeffer. Über die Bedeutung der Begriffe "Maximum und Minimum" in der Variationsrechnung. Math. Ann. 26, (1885), 197-208.

[13] M. Zhang. A relationship between the periodic and the Dirichlet BVP's of singular differential equations. Proc. Royal Soc. Edinburgh 128A (1998), 10991114 . 